\title{
Earlier IV Fluid and Antibiotic Administration with an ED Electronic Sepsis Screening Tool
}

\author{
Nicholas Graff, DO and Robert Nolan, DO \\ Lakeland Health, Saint Joseph, Michigan 49085, USA
}

\begin{abstract}
We set out to overcome barriers previously shown to impede sepsis therapy. Application of a universally standardized therapy model is an ongoing controversy. By taking advantage of the novel and adaptable aspects of a new technology, we predict that the introduction of an electronic health record based sepsis identification tool in the emergency department will aid clinicians in earlier implementation of sepsis directed therapy, namely intravenous fluids and antibiotics. A retrospective cohort study of 3,076 patients with the diagnosis of sepsis was performed. Patient charts were identified for this study who met the criteria of: $\geq 18$ years old; emergency department evaluation; $I C D-9$ code of sepsis, severe sepsis, or septic shock. Data was divided into two treatment groups, pre- and post-best practice advisory (BPA) sepsis identification tool. Our primary outcome was time to intravenous fluids and time to antibiotics administration. The secondary outcome of in-patient all-cause mortality was measured. 1,266 patients were treated prior to, and 1,810 treated after, the BPA implementation with a decrease in time to intravenous fluids from $34 \%$ to $49.9 \%$ of patient receiving treatment in the first $60 \mathrm{~min}$ of emergency department arrival (difference of $15.9 \% ; P<0.05$ ). The time to administration of antibiotics in the first $180 \mathrm{~min}$ of arrival improved from $40.3 \%$ to $56.8 \%$ (difference of $16.5 \% ; P<0.05$ ). Secondarily, we found that in-patient mortality improved from $10.5 \%$ to $7.5 \%$, pre- and post BPA respectively (difference of $3 \% ; P<0.05$ ). Our study has demonstrated that the implementation of an active electronic health record screen tool that alerts clinicians to the possibility of sepsis may improve the time to initiate fluid blouses and antibiotics, and may lead to improved outcomes.
\end{abstract}

Key words: Sepsis, therapy, electronic health record, EHR, SIRS, emergency department, ED, ER.

\section{Introduction}

\subsection{Background}

Since 2002, the Surviving Sepsis Campaign has strived to decrease mortality from severe sepsis and septic shock through awareness, education, improvement of treatments and diagnosis, and implementation of practice based performance improvements aimed at sepsis [1]. In the United States, despite significant advances in the detection and care of these patients, over 1 million cases occur with in-hospital mortality ranging from $14.7 \%$ to $29.9 \%$, with an estimated cost of $\$ 17$ billion nationally [2]. Therapeutic development has centered on the efficacy early-goal directed therapy and its success at reducing mortality as well as health care cost and resources

Corresponding author: Nicholas Graff, research fields: health science, medicine, emergency medicine, electronic health record and sepsis. through this standardized multidisciplinary approach [1, 3-5]. Particularly important in the implementation of sepsis therapy is rapid identification of septic patients in the emergency department [6]. This has been an increasing focus within the realm of emergency department performance improvements and education, specifically in developing standardized sepsis treatment and triage identification protocols [7, 8]. The Center for Medicare \& Medicaid Services has asked for universal standards for sepsis therapy that can be difficult to implement, and do not take into account individual emergency department resources or patient centered care $[9,10]$. The Surviving Sepsis Campaign does not endorse a particular emergency department sepsis screening or identification protocol, nor recommendations for improvement of standardized early goal-directed therapy $[9,10]$. There have been, however, multiple studies that demonstrate empiric improvement of patient outcomes from emergency 
department sepsis screening and management bundles focused at early identification $[11,12]$. It can be very difficult to move away from universal and towards institutional standardization [11, 12]. Many technological, logistical, and institutional barriers have been identified in the initiation of emergency department sepsis performance improvements, with diminished healthcare resources and challenges in the recognition of sepsis as two of the most common burdens [13].

\subsection{Goals of This Investigation}

At Lakeland Health in Southwest Michigan, a community based hospital system, with $>80,000$ annual ED visits, we have taken advantage of the novel and adaptable aspects of an electronic health record (EHR), shown to be effective in early recognition of septic patients by way of computerized alerts [14]. Our study sought to increase awareness of sepsis there by breaking down barriers impeding initiation of sepsis care by using a dynamic identification tool to more broadly screen for sepsis in order to improve detection and treatment. We predict that the use of an electronic identification tool will aid in earlier implementation of sepsis therapy.

\section{Methods}

\subsection{Study Design and Setting}

We performed a retrospective cohort study of patients with sepsis with investigation of the change in time to initiation of sepsis therapy before and after the implementation of a performance improvement best practice advisory (BPA) electronic sepsis screening tool. The institutional review board at Lakeland Health reviewed and approved this study.

Data enrolled in this study was gathered in a retrospective manner from the Epic EHR software of patients' charts who presented to the emergency department at Lakeland Health that were encoded to protect personal and medical information. The emergency department is staffed by emergency residents and midlevel providers who are supervised by board-certified emergency medicine physicians at all times. In February 2012 with an institutional shift from paper charts to EHR the department had undergone education and followed a written standardized protocol for the detection and treatment of sepsis based recommendations developed by Dr. Emanuel Rivers, MD and the Surviving Sepsis Campaign $[1,3]$.

\subsection{Participants}

We included only patients 18 years or older who were evaluated by the emergency department and admitted to the hospital between February 2012 and October 2014 with $I C D-9$ diagnosis codes of sepsis, severe sepsis, septic shock. We also reviewed charts with the ICD-9 diagnosis codes that were recoded as sepsis, severe, sepsis, septic shock for billing to verify that the patient met those criteria. Excluded were patients who were under the age of 18 , transferred to another facility prior to admission, placed in comfort care only and/or hospice care, or had incomplete data (lack emergency department arrival time, intravenous antibiotics and fluids, or mortality data), or did not meet $I C D-9$ coding definition of sepsis, severe sepsis, or septic shock.

\subsection{Intervention}

The primary intervention was the implementation of a BPA at the end May 2013. The BPA is an electronic tool designed to alert clinician of a patient with possible sepsis based on parametric information of a modified systemic inflammatory response syndrome (SIRS) criteria that began with vital signs inputted in the EHR at time of triage, ambulance arrival in the emergency department, and/or any point during the patient's stay as vitals changed or more data became available; for example leukocytosis upon return of initial blood tests. The trigger criteria included: temperature $<36{ }^{\circ} \mathrm{C}$ or $>38{ }^{\circ} \mathrm{C}$; heart rate $\geq 90$ beats per minute; respiratory rate of $\geq 20$ breaths per minute; white blood cell count $<4,000\left(4 \times 10^{9}\right.$ cells $\left./ \mathrm{L}\right)$ 
or $>12,000\left(12 \times 10^{9}\right.$ cells/L $)[15]$. As an electronic tool the BPA was a hard stop in our EHR firing when at least 2 out of 4 SIRS criteria were met. This forced the user to select options, "Accept", at risk for sepsis, or "Cancel", low-risk/does not meet sepsis criteria, from an alert box shown prior to closing the patient chart. If the clinician suspected sepsis was possible a sepsis specific order set containing diagnostic and treatment orders including fluid resuscitation (institutionally using $1 \mathrm{~L} 0.9 \%$ sodium chloride bolus over $1 \mathrm{~h}$ aliquots) and antibiotic recommendations were linked to the BPA and could be opened automatically. Diagnostic and treatment order sets were created by a multidisciplinary team of physicians and pharmacists and are based on the Surviving Sepsis Campaign recommendations $[1,3]$. We were able to track the BPA criteria triggering and use of order sets via the Epic EHR software. Regardless of intervention, all patients were treated similarly by the same emergency medicine and hospitalist team.

\subsection{Methods and Measurements and Outcomes}

Retrospectively, between February 25, 2012 and September 31, 2014, anonymized EHR chart data meeting inclusion criteria was coded and assembled by a single unblinded individual with Crimson Clinical Advantage, a business intelligence tool from The Advisory Board Company. We analyzed the data measuring: age, sex, discharge status, date and time of emergency department encounter, time to intravenous (IV) fluids and IV antibiotics. Data was then placed into two treatment groups using Microsoft Excel and configured with QI Macros, a Microsoft software. We employed five out of the eight strategies to improve the chart review quality per Gilbert, et al [16]. These included using a highly trained individual, who followed well-defined case section and variables, a standardized abstraction process, and monthly meetings to review data quality.

The treatment groups consisted of one treatment group prior to BPA implementation and a second after the BPA was operational. June 1st, 2013 was the agreed upon date when the BPA programming was fully functional after implementation. It was first implemented May 23rd, 2013 with possible errors made. 33 patients from May 23rd through May 31st were included in the final statistical analysis.

We analyzed and inputted the data into Microsoft Excel as follows: emergency department arrival time was defined as the time of registration within the EHR. Door to IV and Door to antibiotic times were both estimated based on an EHR time stamp of nursing verified administration from the difference in time from emergency department arrival. Institutional policy is to electronically verify initiation of any treatment immediately prior to administration, or after in the event of emergent need for treatment. Patient discharge status data was reassembled into binominal data as discharged living vs. deceased. Patients discharged after hospital admission defined as living included: discharge to home; transferred to secondary inpatient facility, long term cared nursing facility, or assisted living facility. Data was also collected concerning patients' age and sex in order to identify possible benefit to at risk populations, such as those over the age of 65 .

\section{Outcomes}

Our primary outcome measured was time to treatment with fluids and antibiotics as a marker of recognition of sepsis. We chose to follow both time to intravenous fluids and antibiotic administration due to their proven correlation to mortality from sepsis with positive outcome previously seen when started within 3 hours of sepsis identification [1,7]. The secondary outcome of in-patient mortality was measured as a binomial endpoint, living vs. deceased at time of discharge. The Centers for Medicare and Medicaid Services definition of mortality based on the Yale New Haven Health Services Corporation/Center for Outcomes Research and Evaluation for standardization $[17,18]$. In-hospital mortality was chosen due to our 
inability to assess 30 day mortality of discharged patients.

\subsection{Analysis}

The primary data analysis was performed using Statistical Package for the Social Sciences produced by International Business Machines Corporation. Categorical data were reported using $c h i^{2}$ tests to determine existing differences between groups. A group was defined as the pre-BPA versus the post-BPA data pertaining to a specific outcome and timeframe, such as, time to intravenous fluids prior to $60 \mathrm{~min}$. Comparisons were not statistically calculated between groups for the primary outcomes. $P$ value of less than .05 was chosen to represent statistical significance. Changes in the total number of subjects from the primary and secondary outcomes can be related to lack of charting information when reviewing relevant charts for data.

\section{Results}

The study contained data on a total of 3,077 patients. 2,458 patient charts contained data related to door to intravenous fluid time with 946 charts prior to BPA and 1,512 after BPA initiation, as demonstrated in Figure 1. The administration of intravenous fluids prior to 60 min after emergency department arrival occurred in 322 prior to, and 753 after BPA initiation, 34\% and $49.9 \%$ of the respective totals (difference of $15.9 \%$; $P<$ 0.05). After $120 \mathrm{~min}$ of arrival in the emergency department 316 patients received intravenous fluids prior to BPA and 265 received them after BPA initiation, $33.4 \%$ and $17.5 \%$ of the respective totals (difference of $15.9 \%$; $P$ of $<0.05$ ). There was no statistical significance in door to intravenous fluid administration time between 60 and $120 \mathrm{~min}$ from emergency department arrival between groups.

The outcome of door to intravenous antibiotics administration was recorded in 2,760 patient charts with 1,145 treated prior to BPA and 1,615 after BPA initiation, demonstrated in Figure 1. Of this, 461 patients received antibiotics from emergency department arrival to 180 min prior to BPA and 918 patients after BPA initiation resulting in $40.3 \%$ and $56.8 \%$ of the respective totals (difference of $16.5 \%$; $P<0.05)$. After $180 \mathrm{~min}$ there was no statistical significance between groups.

The secondary outcome of in-patient mortality was recorded in 3,076 patient charts with 1,266 receiving sepsis therapies prior to BPA and 1,810 after BPA initiation resulting in primary in-patient mortality of $10.5 \%$ and $7.5 \%$, respectively (difference of $3 \% ; P<$ $0.05)$.

There was no difference when controlling for sex between groups. When controlling for age, 18-64 years of age versus 65 years and older, there was a significance found in the 65 and older group with $12.4 \%$ in-patient mortality prior to BPA and $8.9 \%$ after BPA initiation (difference of $3.5 \% ; P<0.05$ ). Differences in age and sex between groups demonstrated in Table 1.

This includes a new class of residents July 1st of each year and three attending physicians who joined the group in July 2013.

\section{Discussion/Limitations}

There are several confounding factors that could have influenced the outcome of this study that we could not control. While we show a strong correlation with the BPA being associated with earlier intravenous fluids and antibiotics there could be outside factors that could have contributed to the improvements seen. We are not able to state causation, however, we can correlate treatment of sepsis at an earlier time during patient care after our BPA identification tool was launched within the Epic, EHR, software. It is possible that education on and discussion of sepsis alone contributed to the improvement of in-patient morality. As noted in the over 65, age group, there was mortality benefit of $0.5 \%$ greater than those $18-64$ years old. We cannot conclude that our study suggests that there is improved benefit to those potentially at higher risk for 


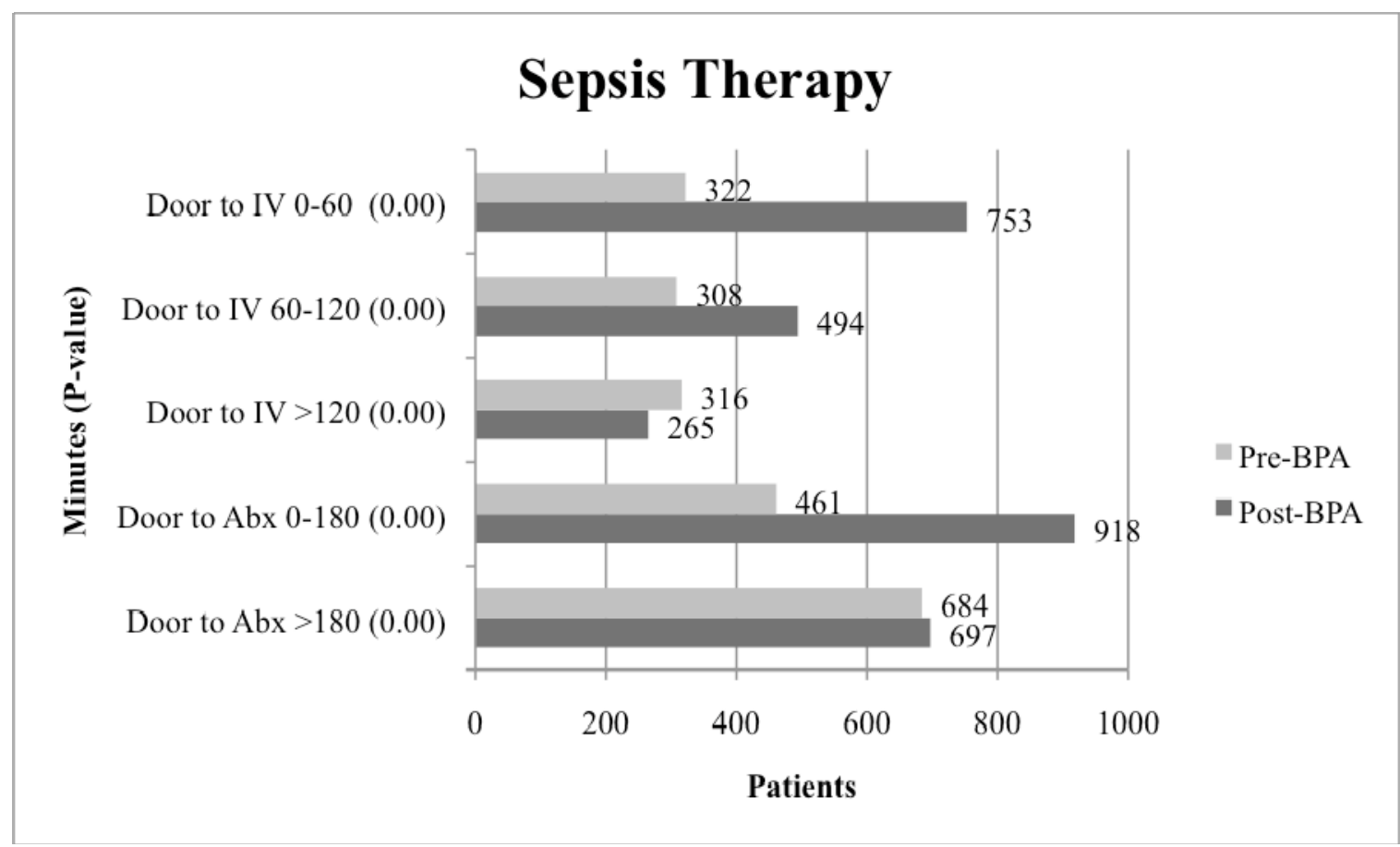

Fig. 1 Time in minutes from ED arrival (Door) to IV fluids (IV) and antibiotics (Abx) pre- and post-BPA. All $P$-values were calculated less than 0.001 .

Table 1 Age and sex distribution of overall mortality pre- and post- BPA.

\begin{tabular}{lllll}
\hline Age (years) & $\begin{array}{l}\text { \# Patients } \\
\text { Discharged Alive }\end{array}$ & Expired & Total (percent of total) & $P$-value \\
\hline $18-64$ & & & & 0.184 \\
Pre-BPA & $423(92.4 \%)$ & $35(7.6 \%)$ & $458(100 \%)$ & $786(100 \%)$ \\
Post-BPA & $741(94.3 \%)$ & $45(5.7 \%)$ & $793(100 \%)$ & 0.018 \\
$65+$ & & & $1,007(100 \%)$ & \\
Pre-BPA & $695(87.6 \%)$ & $98(12.4 \%)$ & & \\
Post-BPA & $917(91.1 \%)$ & $90(8.9 \%)$ & $575(100 \%)$ & 0.033 \\
Sex & & & $846(100 \%)$ & \\
Male & & $64(11.1 \%)$ & \\
Pre-BPA & $511(88.9 \%)$ & $66(7.8 \%)$ & $691(100 \%)$ \\
Post-BPA & $780(92.2 \%)$ & $69(10.0 \%)$ & $964(100 \%)$ \\
Female & & $69(7.2 \%)$ & \\
Pre-BPA & $622(90.05)$ & & \\
Post-BPA & $895(92.8 \%)$ & & \\
\hline
\end{tabular}

mortality from sepsis versus causing harm to those under the age of 65 .

We acknowledge the external confounding factors and their many possible effects on our outcomes. We did not control for individual provider education or update in sepsis treatment guidelines in 2013. In future studies it may be important to standardize education. We were not able to fully control for standardize treatment practices between nursing and/or clinical providers beyond the standardized sepsis treatment bundle within our EHR, that the providers were not required, but encouraged to use. It was assumed that providers were following standard of care based on Surviving Sepsis Campaign's guidelines, however we cannot say this for certain in all cases. We were unable to assess for out-of-hospital mortality and in future studies we plan on developing a modality to track 30-day mortality of septic patients. 
Additionally, we did not identify total number of patients who triggered the BPA alert that did not have the diagnosis of sepsis. However, we do not feel that this would have been relevant to our clinical question.

While our hospital system's modified sepsis protocol may not be generalizable to other institutions, recent studies (ARISE and ProCess trials) have shown that a tight sepsis protocol is not necessary to improve survival, but that earlier recognition and treatment of sepsis may be $[11,12]$. We believe that this is why improvement in mortality was demonstrated.

\section{Conclusions}

Our study has demonstrated the effective implementation of an electronic health record based BPA identification tool in the emergency department. We demonstrated with great likelihood that implementation of our BPA in conjunction with education and the electronic health record led to earlier initiation of both IV fluids and antibiotics from time of emergency department arrival, and may have contributed to reduction in mortality.

\section{Reference}

[1] Dellinger, R. P., Levy, M. M., Rhodes, A., Annane, D., Gerlach, H., and Opal, S. M., et al. 2013. "Surviving Sepsis Campaign: International Guidelines for Management of Severe Sepsis and Septic Shock, 2012." Intensive Care. Med. 39 (2): 165-228.

[2] Gaieski, D. F., Edwards, J. M., Kallan, M. J., and Carr, B. G. 2013. "Benchmarking the Incidence and Mortality of Severe Sepsis in the United States." Crit. Care. Med. 41 (5): 1167-74.

[3] Rivers, E., Nguyen, B., Havstad, S., Ressler, J., Muzzin, A., and Knoblich, B., et al. 2001. "Early Goal-directed Therapy in the Treatment of Severe Sepsis and Septic Shock." N. Engl. J. Med. 345 (19): 1368-77.

[4] Rivers, E. P., Coba, V., and Rudis, M. 2009. "Standardized Order Sets for the Treatment of Severe Sepsis and Septic Shock." Expert Rev. Anti. Infect. Ther. 7 (9): 1075-9.

[5] Castellanos-Ortega, A., Suberviola, B., García-Astudillo, L. A., Holanda, M. S., Ortiz, F., and Llorca, J., et al. 2010. "Impact of the Survivng Sepsis Campaign Protocols on Hospital Length of Stay and Mortality in the Septic Shock Patients: Results of a Three-year Follow-up
Quasi-experimental Study." Crit. Care Med. 38 (4): 1036-43.

[6] Nguyen, H. B., Rivers, E. P., Abrahamian, F. M., Moran, G. J., Abraham, E., and Trzeciak, S., et al. 2006. "Severe Sepsis and Septic Shock: Review of the Literature and Emergency Department Management Guidelines." Ann. Emerg. Med. 48 (1): 54-e1.

[7] Levy, M. M., Dellinger, R. P., Townsend, S. R., Linde-Zwirble, W. T., Marshall, J. C., and Bion, J., et al. 2010. "The Surviving Sepsis Campaign: Results of an International Guideline-based Performance Improvement Program Targeting Severe Sepsis." Intensive Care Med. 36 (2): 222-31.

[8] Levy, M. M., Pronovost, P. J., Dellinger, R. P., Townsend, S., Resar, R. K., and Clemmer, T. P., et al. "Sepsis Change Bundles: Converting Guidelines into Meaningful Change in Behavior and Clinical Outcome." Crit. Care Med. 32 (11): 595-7.

[9] Jones, A. E., and Puskarich, M. A. 2014. "The Surviving Sepsis Campaign Guidelines 2012: Update for Emergency Physicians." Ann. Emerg. Med. 63 (1): 35-47.

[10] Mouncey, P. R., Osborn, T. M., and Power, G. S., et al. 2015. "Trial of Early, Goal-directed Resuscitation for Septic Shock." N. Engl. J. Med. 372: 1301-11.

[11] Lilly, C. M. 2014. "The ProCESS Trial-a New Era of Sepsis Management.” N. Engl. J. Med. 370 (18):1750-51.

[12] Delaney, A. P., Peake, S. L., Bellomo, R., Cameron, P., Holdgate, A., and Howe, B., et al. 2013. "The Australasian Resuscitation in Sepsis Evaluation (ARISE) Trial Statistical Analysis Plan." Crit. Care Resusc. 15 (3): 162-71.

[13] Carlbom, D. J., and Rubenfeld, G. D. 2007. "Barriers to Implementing Protocol-based Sepsis Resuscitation in the Emergency Department-Results of a National Survey." Crit. Care Med. 35 (11): 2525-32.

[14] Kurczewski, L., Sweet, M., McKnight, R., and Halbritter, K. 2015. "Reduction in Time to First Action as a Result of Electronic Alerts for Early Sepsis Recognition." Crit. Care Nurs. Q. 38 (2): 182-7.

[15] Bone, R. C., Balk, R. A., Cerra, F. B., Dellinger, R. P., Fein, A. M., and Knaus, W. A., et al. 1992. "Definitions for Sepsis and Organ Failure and Guidelines for the Use of Innovative Therapies in Sepsis." The ACCP/SCCM Consensus Conference Committee American College of Chest Physicians/Society of Critical Care Medicine. Chest 101 (6):1644-55.

[16] Gilbert, E. H., Lowenstein, S. R., Koziol-McLain, J., Barta, D. C., and Steiner, K. 1996. "Chart Reviews in Emergency Medicine Research: where are the Methods?" Ann. Emerg. Med. 27 (3): 305-8.

[17] Quality Net: The Centers for Medicare \& Medicaid Services: Mortality Measures. [Internet]. [place 
unknown:publisher unknown] [updated 2015 Mar]. Available from: http://www.qualitynet.org/dcs/ContentServer?c=Page\&pa gename $=$ QnetPublic\%2FPage\%2FQnetTier2\&cid $=12287$ 63452133 .

[18] Krumholz, H. M., Brindis, R. G., Brush, J. E., Cohen, D. J., Epstein, A. J., and Furie, K., et al. 2006. Standards for
Statistical Models used for Public Reporting of Health Outcomes: an American Heart Association Scientific Statement from the Quality of Care and Outcomes Research Interdisciplinary Writing Group: cosponsored by the Council on Epidemiology and Prevention and the Stroke Council. Endorsed by the American College of Cardiology Foundation. Circulation 113 (3): 456-62. 